\title{
Comparing Holmium (Ho): YAG Laser with Pneumatic Lithoclast for Treatment Efficacy of Ureteric Stones
}

Asadullah', Salman el Khalid², Waqar Ahmed Memon ${ }^{3}$, Ali Haider', Adnan Siddiq Awan ${ }^{5}$, Muhammad Bilal Quddus $^{6}$ 'Department of Urology, Sandeman Provincial Hospital, Quetta, '2Department of Urology, the Kidney Center, Postgraduate Training Institute, Karachi, ${ }^{3}$ Department of Urology, Liaquat University of Medical and Health Sciences (LUMHS) Jamshoro, ${ }^{4}$ Department of Urology, Hamdard University Hospital, Karachi, ${ }^{5}$ Department of Urology, PAF Hospital Masroor, Karachi, 'Victoria Hospital Blackpool, Blackpool, United Kingdom.

\section{ABSTRACT}

Background: Holmium YAG (yttrium-aluminum-garnet) laser, a comparatively new technique, can clear all types and sizes of stones and is only being used in two institutes of Karachi, Pakistan. The study aimed to compare pneumatic lithoclast and holmium YAG laser, to evaluate stone-free rate (SFR), postoperative complications, operative duration, and effectiveness.

Methods: This cross-sectional research included 60 patients with age 16-65 years having ureteric stones, reporting to kidney center, Urology Department, Karachi, Pakistan. CT Scan of Kidneys, Ureter, and Bladder (KUB) were used to record stone size, laterality, and location within the ureter. Patients were divided into two clusters of 30 each, group A (Ho: YAG laser) and group B (pneumatic Lithoclast) having 0.5 to $02 \mathrm{~cm}$ of size ureteric stones. An Independent two-sample "t" test was used to assess the difference for the continuous variables. A p-value of $\leq 0.05$ was considered statistically significant.

Results: Stone sizes distribution was $1.3 \pm 0.3 \mathrm{cms}$ and $1.4 \pm 0.3 \mathrm{cms}$ for $A$ and $B$ groups respectively $(p=0.8)$. The insignificance of the $p$-value demonstrated no substantial divergence between both groups and stone sizes. Clearance from the proximal ureter was noted $26(84.6 \%)$ in group A and $41.7 \%$ in group $B$ with $(p<0.05)$. A reduced lithotripsy activation period of $30.8 \pm 3.7$ mins was associated with stone size $(p<0.05)$.

Conclusion: Holmium: YAG Laser had better stone-free rate (SFR), with $84 \%$ clearance than pneumatic. Improved and effective clearance reduces the risk of residual stones within a lesser time, required for getting back to normal life routines.

Keywords: Lasers; Ureteric Stones; Ho: YAG Laser; Pneumatic Lithoclast; Kidney Stones.

\author{
Corresponding Author: \\ Dr. Asadullah \\ Department of Urology, \\ Sandeman Provincial Hospital, Quetta, Pakistan. \\ Email: a4asad21@hotmail.com \\ https://doi.org/10.36283/PJMD1 1-1/002
}

How to cite: Ullah A, el Khalid S, Memon WA, Haider A, Awan AS, Quddus MB. Comparing Holmium (Ho): YAG Laser with Pneumatic Lithoclast for Treatment Efficacy of Ureteric Stones. 2022;11(1): 4-10. doi: 10.36283/PJMD11-1/002 


\section{INTRODUCTION}

Renal stones are prevalent worldwide, tormenting as the third most commonly occurring renal disease after Urinary tract infection and prostate problems such as benign prostate hyperplasia, approximately $20 \%$ incidence rates have been recorded with higher frequencies in Asian countries like India, Pakistan, Afghanistan, and Nepal. Pakistan is known to be geographically situated on the Afro-Asian stone belt, which makes Pakistani population incidence rates higher than neighboring countries. The reason for increased prevalence varies between the geographical placement of Pakistan along with nutritional deficiencies and delayed health-seeking behavior of population ${ }^{1,2}$.

Renal physiology tends to pass out the small renal stone with a maximum of $0.4 \mathrm{~cm}$ of size, any stone larger than $0.4 \mathrm{cms}$ could be stuck in the kidney or ureter, causing severe pain, hydronephrosis, hematuria, and obstructive uropathy. In the $0.4 \mathrm{cms}$ size stones, $70 \%-90 \%$ are passed without any adverse outcome and sometimes cause mild and tolerable pain to the patient ${ }^{3,4}$. The size of renal stones determines the requirement of intervention in urology; smaller stones with higher chances of smooth passage do not usually require any intervention the best method is to wait. The urology surgeons decide after complete evaluation of stone size, site, and location whether any kind of intervention is necessary or not. The protocols to manage ureteric calculi have been progressed in the past few decades from percutaneous nephrolithotomy for pushed back stones, extracorporeal shockwave lithotripsy (ESWL) to ureteroscopy (URS). Especially in managing the middle and distal area, ureteric calculi have been changed enormously after the introduction of assorted ureteroscopy methodologies and lithotripters. From the list of many procedures, two lithotripters are known as most effective in the present era, an old and trusted Pneumatic lithoclast and a new, modified, and efficient holmium YAG laser $^{5,6}$. The published literature is supportive of laser with an efficacy of $92 \%$ for laser and $82.1 \%$ for lithoclast. Some researchers assessed the mean difference of stone fragmentation time and stone-free rates amid both lithotripters and specified higher fragmentation and reduced lithotripsy period in laser ${ }^{7,8}$.

Pneumatic lithoclast is more frequently used by urologists as they are known to be easy to use, install, and comparatively cost-effective. Pneumatic lithoclast is a cheap treatment option for patients. However, complications like increased retropulsion of stone in kidney especially while fragmenting larger calculis. Coagulation properties along with vaporization of tissues, holmium laser has come forward as a superior substitute to pneumatic lithotripter. Holmium YAG laser can clear all types and sizes of stones. However, its elevated cost and preservation cost restricts its use in developing countries like Pakistan. Research with 102 participants indicated $50 \%$ efficacy of SFR with pneumatic lithoclast for ureteric stones?.

With the arrival of innovative versions of lithotripters, urologists' job has been made easy with decreased chances of retropulsion and resulting morbidity. Urology institutes of Pakistan pneumatic lithoclast were tried and tested modality for ureteric calculi for decades, Holmium Yag laser is comparatively new and has only been used in two institutes of Karachi, Pakistan. The usage of holmium YAG laser requires highly experienced surgeons, increased cost, and maintenance, which is not fitting for small capacity urology centers. The study results will be a beneficial addition to the literature for any difference present in Stone Free Rates, post-operative complication, smaller operative span, duration of lithotripter activation (minutes), and total operative time difference within both modalities. This study aimed to compare the efficacy of pneumatic lithoclast and holmium YAG laser in the treatment of ureteric calculi measuring $<2.0 \mathrm{cms}$ and to evaluate Stone-free rate (SFR), postoperative complications, and operative duration.

\section{METHODS}

This is a prospective study with a cross-sectional research design. The patient data $(n=60)$ was obtained from the kidney center, Urology Department, Karachi Pakistan. The data were collected for four months starting from December 2020. Upon ethical approval from the institutional ethical review committee with reference \# 48-URO-070223. A purposive, non-probability sampling technique was used, patients reporting in health care institutes ranging from 16-65 years age group presented with ureteric stones within four months were enrolled in the study. CT KUB was used as a diagnostic tool to confirm stone size, laterality, and location within the ureter. Only 0.5 to $02 \mathrm{cms}$ of size ureteric stones were included in the study. Patients with large stone sizes, multiple stones, and gross hydronephrosis were excluded from the study. After signing the consent, the demographic details, such as age, weight, gender, relevant history about co-morbidities and previous renal stone history, and laboratory investigations were recorded.

Computer-based software was used for randomization to minimize the confounding factors; participants were randomly sorted into two groups. Group $A$ included the patients who got Holmium YAG laser modality while Group B patients got Pneumatic lithoclast as a modality. Fragmentation time and SFR on the operation table were documented. Patients were asked to visit OPD after 7 days of procedure for follow-up; imaging modalities such as 
X-rays and ultrasound of Kidneys, Ureters, and Bladder (KUB) areas were performed to assess the presence of residual stones and evaluate stone-free rates, and confirmation of any residual calculi or retropulsion calculi on follow up radiological reports. No retropulsion with complete clearance on the $7^{\text {th }}$ follow-up day of surgery was measured as effective surgery. All the collected data were entered in version 20 of the statistical package for social science (SPSS). SPSS was used for the analysis of data; descriptive variables such as age, weight, gender was analyzed, and results were measured in mean and standard deviation values. Stone characteristics including size and operative details with lithotripsy time, serum creatinine was mentioned as meanwhile stone location and site were reported in frequencies. An independent two-sample " $f$ " test was performed to evaluate the difference for the continuous variables. A p-value of 0.05 or less was measured as statistically significant.

\section{RESULTS}

The sample size $n=60$ of the study having age $16-65$ years with the predominant age group was 30-35 years. Patients were arranged in equal numbers in two clusters (groups), in group A 30 patients went through Ho: YAG laser for stone fragmentation of ureter, similarly, group B participants had pneumatic Lithoclast in 30 patients. To eliminate the chances of biased results, the significance of the data was measured. The mean age of group A members was $34.8 \pm 4.0$ years and $34.5 \pm 3.4$ years in group B. The gender allocation of groups was $36.7 \%$ male and $63.3 \%$ female, and $40 \%$ male and $60 \%$ female for Laser and Pneumatic. The statistics were significant, $p$-value $<0.05$. The dominance of the female population in our study is diverse from other referenced studies. The serum creatinine was calculated in mean \pm standard deviation, representing an outcome of $1.0 \pm 0.9$ and $1.2 \pm 1.1$ for the Laser and Pneumatic groups respectively, with a significant p-value $<0.05$.

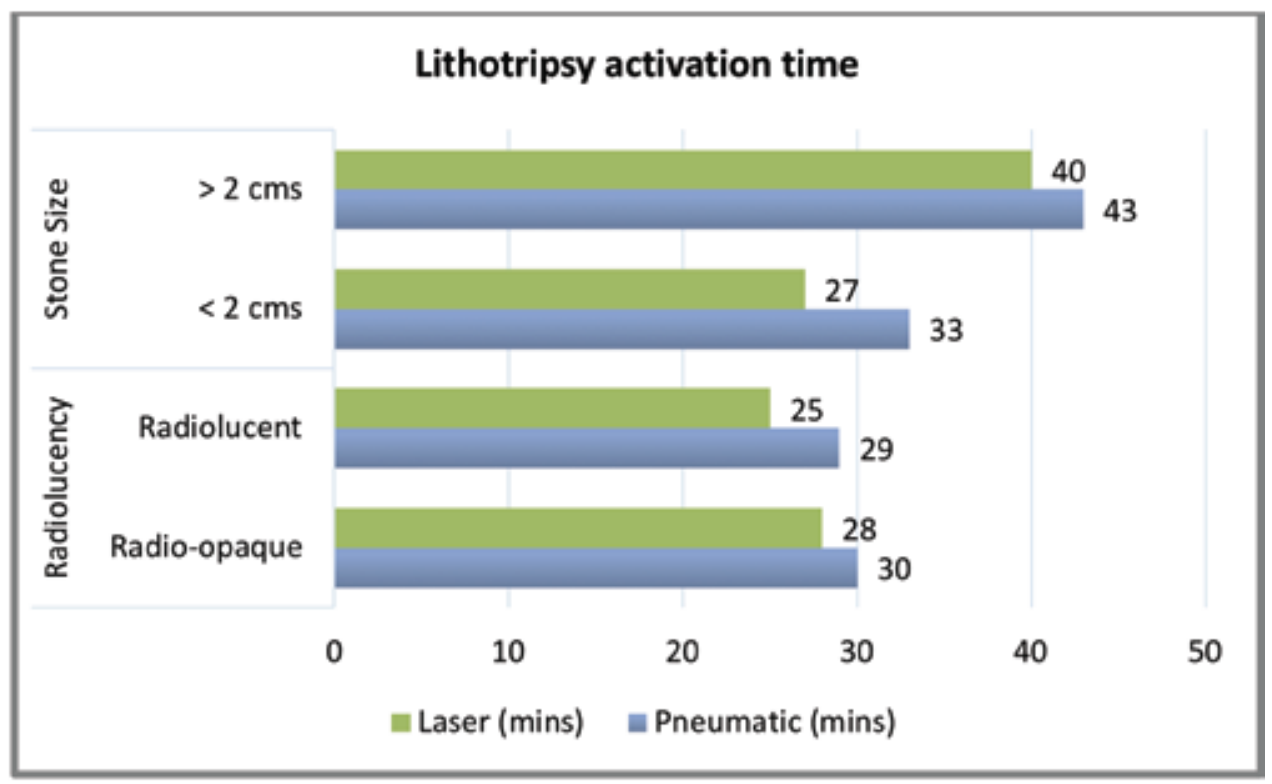

Figure 1: Lithotripsy activation time associated with radiolucency and stone size (group A - Holmium (Ho): YAG Laser, group B - Pneumatic Lithoclast).

Stone sizes were measured $<2 \mathrm{cms}$, the distribution of stone size was $1.3 \pm 0.3 \mathrm{cms}$ and $1.4 \pm 0.3 \mathrm{cms}$ (Figure 1) for $A$ and $B$ groups respectively with $p$-value $=0.8$, the insignificance demonstrated no substantial divergence between both groups and stone sizes. The site of stone was dominant on the left side, like other renal stone studies with higher prevalence rates of stones on the left side. The position of stone was calculated in groups demonstrating $40 \%$, $16.7 \%$, and $43.3 \%$ in proximal, mid, and distal ureteric stones of Group A participants. The results of group B were $33.3 \%, 16.7 \%$, and $50 \%$ in proximal, mid, and distal ureter respectively, with a significant $p$-value of $<0.05$. The activation time of the lithotripter in group A was $32.3 \pm 3.8$ mins, while in group B $30.8 \pm 3.7 m i n s$. Upon analyzing the association of stone size with lithotripter activation time the outcome was $30.2 \pm 3.6$ mins and $28.6 \pm$ 3.6 mins in groups A and B respectively with a $p$-value of $<0.05$ as shown in Table 1. 
Table 1: Details of demographics, lithotripsy activation time, and distribution of stone location within ureter with stone clearance.

\begin{tabular}{|c|c|c|c|c|}
\hline \multicolumn{2}{|l|}{ Variables } & \multirow{2}{*}{$\begin{array}{c}\text { Laser Group (A) } \\
27(90 \%)\end{array}$} & \multirow{2}{*}{$\begin{array}{c}\text { Pneumatic Group (B) } \\
22(73.3 \%)\end{array}$} & \multirow{3}{*}{$\frac{p \text {-Value }}{0.09}$} \\
\hline \multirow{2}{*}{ Gender } & Male & & & \\
\hline & Female & $3(10 \%)$ & $8(26.7 \%)$ & \\
\hline \multirow{3}{*}{ Location in ureter } & Proximal & $12(40 \%)$ & $10(33.3 \%)$ & \multirow{3}{*}{0.9} \\
\hline & Mid & $5(16.7 \%)$ & $5(16.74 \%)$ & \\
\hline & Distal & $13(43.3 \%)$ & $15(50 \%)$ & \\
\hline \multirow{3}{*}{ Clearance results } & Complete Clearance & $26(84.6 \%)$ & $19(63.3 \%)$ & 0.03 \\
\hline & Residual stone & $1(3.3 \%)$ & $3(10 \%)$ & 0.3 \\
\hline & Retropulsion & $3(10 \%)$ & $8(26.7 \%)$ & 0.09 \\
\hline \multicolumn{2}{|l|}{ Age } & $36.8 \pm 10.68$ & $37.2 \pm 14.57$ & 0.9 \\
\hline \multicolumn{2}{|l|}{ Stone size } & $1.35 \pm 0.35$ & $1.45 \pm 0.31$ & 0.82 \\
\hline \multicolumn{2}{|c|}{ Lithotripsy activation Time } & $30.8 \pm 3.71$ & $32.3 \pm 3.8$ & 0.1 \\
\hline \multicolumn{2}{|c|}{ Lithotripsy activation associated with stone size } & $28.6 \pm 3.6$ & $30.2 \pm 3.6$ & 0.05 \\
\hline
\end{tabular}

In addition, $84.6 \%$ clearance was noted in group $\mathrm{A}$ from the proximal ureter, $41.7 \%$ clearance from group $B$ was reported from the same location with $<0.05$ p-value. Ho: YAG Laser was vastly competent in the clearance of mid and distal ureter stones as well. Division of lithotripter activation time with the allocation of radiolucency, calculi dimension, and locations of stone within groups associated with lithotripsy activation time was signified in (Figure 2).

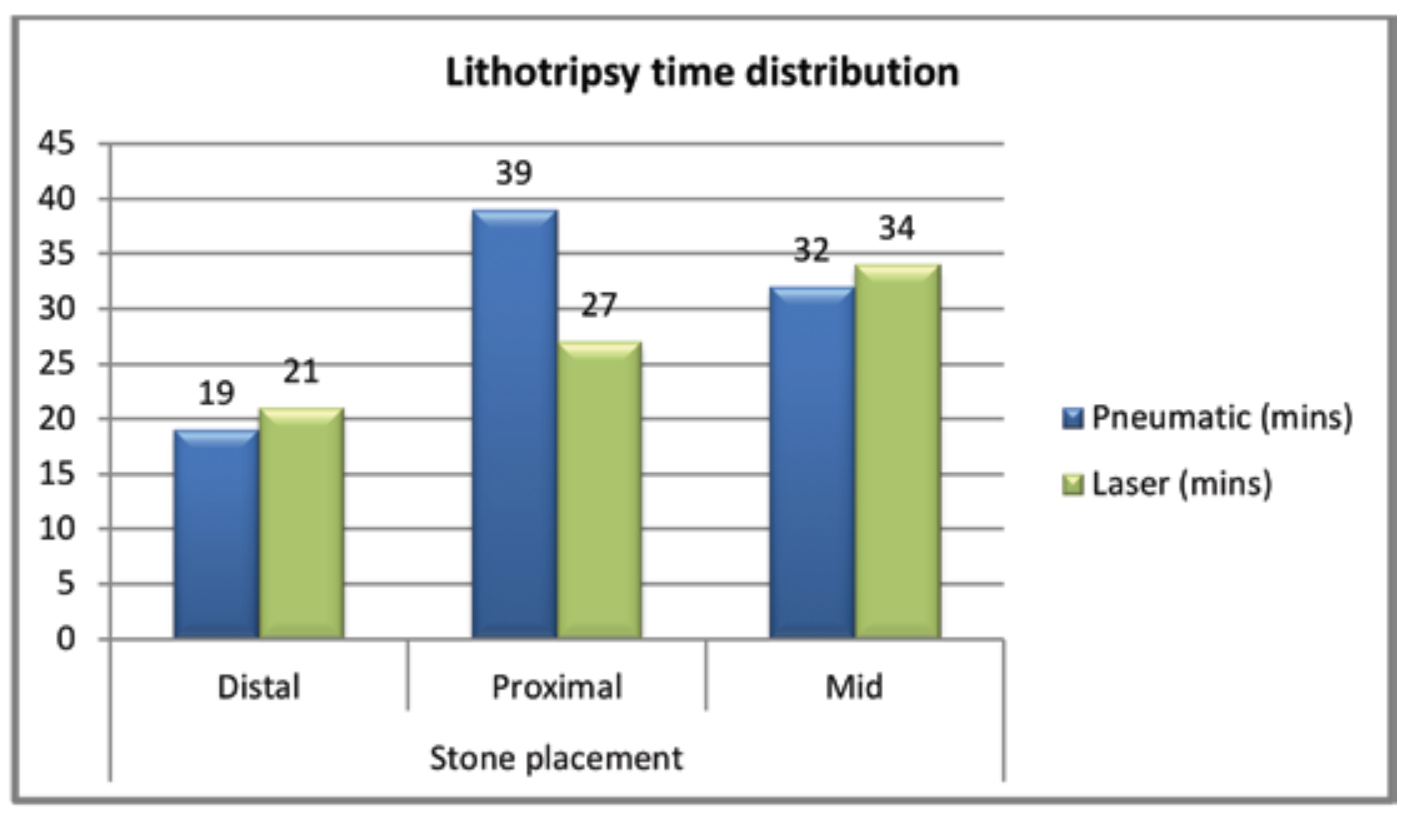

Figure 2: Lithotripsy time distribution associated with a stone location within the ureter.

Age, gender, and size of calculi were not established to be an interpreter for clearance of stone. The difference in lithotripter activation time and total operative time from both groups is comparable as only one surgeon performed all the cases, eradicating the surgeon's learning curve delays and error. Double J stent was inserted in all patients after surgery to avoid ureteric spasm and improve stone passage, Double J stent was removed after 3 weeks of surgery. Accumulated operative time, catheterization, hospital stay, stone-free rates, and complication rates of group A and Group B are mentioned in Table 2. 
Table 2: Operative details within Group A and Group B.

\begin{tabular}{|l|c|c|c|}
\hline Variables & Laser Group (A) & Pneumatic Group (B) & $\mathbf{p}$-Value \\
\hline Total operative Time (mean \pm SD) & $48.5 \pm 9.2$ & $56.4 \pm 11.1$ & 0.03 \\
\hline Catheterization $\mathbf{n}$ (\%) & $80 \%(24)$ & $90 \%(27)$ & 0.07 \\
\hline Hos pitalization days (mean $\mathbf{\text { SD } )}$ & $1.3 \pm 0.9$ & $1.5 \pm 0.6$ & 0.1 \\
\hline Stone free rate $\mathbf{n}$ (\%) & $26(86.7 \%)$ & $19(63.3 \%)$ & 0.03 \\
\hline Complication rate $\mathbf{n}(\%)$ & $5(16.6 \%)$ & $11(36.6 \%)$ & 0.04 \\
\hline
\end{tabular}

\section{DISCUSSION}

Managing ureteric stones have been transformed radically in a few years, including affirming theories indicating the beneficial effect of medical expulsion therapies on small-sized $(<0.8 \mathrm{cms})$ distal ureteric stones, to different lithoclast used by urologists while performing URS. This study signifies the efficacy of two major modalities used in Pakistan's best urology institutes to eradicate ureteric stones. The results specified that Ho: YAG Laser has better Stone Fee Rates, with $84 \%$ clearance than pneumatic with a reduced lithotripsy activation period of $30.8 \pm 3.7 \mathrm{~min}$. Although, some other studies specified improved stone-free rates $88 \%$ and smaller operation duration $7.86 \pm$ $3.25 \mathrm{~min}$ with pneumatic lithoclast the variance between both pieces of research possibly because of stone size and arrangement facilitate lithotripter to acquire extra time in crumbling the calculi ${ }^{10,11}$.

The Ho: YAG laser fragment the stones in minute dusting constituent parts, trouble-free to pass lacking soreness or other undesirable results ${ }^{12,13}$. Our study outcomes demonstrated the elevated frequency of female patients with $60 \%$ and $63.3 \%$ in the laser versus pneumatic group. Distal ureteric stones were maximum in numbers with $50 \%$ frequency in the laser group and $43.4 \%$ frequency in the pneumatic group and different researches indicated comparable results ${ }^{14,15}$. For calculating lithotripsy, duration there's no standardized method, an RCT compared pneumatic and Ho: YAG Laser and evaluate complete operation time mentioning increased duration in the pneumatic group by $18 \pm 3.4$ difference between the group. The lithotripter activation time of this study is $32.3 \pm 3.8$ versus $30.8 \pm 3.7$ in pneumatic and laser group respectively with a $p$-value of 0.1 , which is like referenced researches ${ }^{16,17}$. Upon dividing the procedure time with stone characteristics to evaluate the variation, the location of stones showed elevated lithotripsy time in proximal ureter with a difference of 10 minutes. Another local study indicated higher clearance rates in proximal ureteric stones by pneumatic modality with $52.6 \%$ clearance and overall stone-free rates of $88.5 \%{ }^{18}$.

While a study with a similar sample size of 60 patients evaluated stone clearance rates and resulted in $92 \%$ stone-free rates with pneumatic lithoclast in proximal ureter stones ${ }^{19}$. Rigid stones revise their position during the pneumatic lithoclast, the location changes during the procedure make it difficult for the urologist to emphasize on stone and break it. At the same time as affirmed in several pieces of research Ho: YAG Laser carries superior usefulness weigh against pneumatic modality with improved stone-free rates and decreased operative duration ${ }^{14,20}$. This study results indicated $86.7 \%$ versus $63.3 \%$ stone clearance on follow-up radiological investigations such as ultrasound KUB and X-rays KUB, in pneumatic and laser groups respectively. Retropulsion had only been reported in proximal ureter calculi ${ }^{20}$.

Reported results indicate improved chances of retropulsion in pneumatic lithoclast modality with $10 \%$ of retropulsion in the Ho: YAG Laser and $26.7 \%$ in the Pneumatic lithoclast group. Due to the best possible results, the Ho Yag laser should be the first choice as a fragmentation modality specifically in proximal ureter stones. Similarly, the pneumatic lithoclast method is more effective in the fragmentation of the middle and inferior stones of the ureter ${ }^{14,21}$. A randomized control trial indicated smaller lithotripsy duration of pneumatic lithoclast with the difference of 10-15 mins approximately, while clearance differences for ureteric stones were recorded higher in the laser group with $79.3 \%{ }^{22}$. Another study evaluated a larger group of 100 patients in each category, the results of this comparative study indicated higher laser lithotripsy activation duration for renal stones with the difference of 8-12 minutes with pneumatic lithoclast, the study groups mentioned equal stone sizes and similar location. While stone-free rates were recorded as improved in the laser group ${ }^{23}$.

The duration of lithotripter activation differs in almost every referenced research; the reason could be the composition and size of the targeting stone ${ }^{24}$. Another study indicated lower operative duration and higher clearance rates in the holmium YAG laser group as compared to pneumatic ${ }^{25}$. Although the safety profile of Ho YAG: Laser is improved as compared to pneumatic lithotripter, the choice of using this modality is still doubtful for proximal ureteric stones. Upon assessing the stone-free rates within proximal ureter, the efficacy of pneumatic lithoclast out shadowed the Ho: YAG laser and specified improved stone-free rates with 
fewer complications. Surgeons' knowledge is suggestively associated with increased success and decreased complications in ureteroscopic laser lithotripsy with the holmium laser. The association of gender, stone size, and radiolucency had no significant effect upon stone-free rates, although the location of stones within the ureter specified by many other studies indicated retropulsion in proximal ureteric stones. The limitation of the study was a smaller number of study participants, a multi-center study with improved sample size is required to assess the accurate outcomes, and comparing advantages as well as complications in all modalities used to treat ureteric stones is required. The comprehensive categorization of study participants with co-morbidities, stone size, location and reported post-operative complications may help in establishing a valuable piece of literature to defend the best modality for the ureteric stone.

\section{CONCLUSION}

Ho: YAG laser was found more effective than the former modality of Pneumatic lithoclast for eradicating ureteral stones. However, Pneumatic lithoclast success rates were higher in proximal ureter stones. For mid ureter and distal ureter stones Laser Ho: YAG was comparable with Pneumatic lithoclast efficacy. Vigilant assessment of stone location is crucial for the choice of lithotripter for many urology surgeons, to obtain maximum fragmentation of ureteral stones, reduced lithotripsy time, and complete operative timings.

\section{ACKNOWLEDGEMENTS}

The authors would like to acknowledge the Department of Urology staff for their support in data collection.

\section{CONFLICT OF INTERESTS}

The authors declared no conflict of interest.

\section{ETHICS APPROVAL}

The Institutional Ethical Review Board had approved the study with reference \# 48-URO-070223.

\section{PATIENT CONSENT}

Consents were obtained from all the participants of the study.

\section{AUTHORS' CONTRIBUTION}

$\mathrm{AU}$ proposed the research question, created a questionnaire, and wrote the manuscript. SEK supervised the research. WAM maintained data transparency and confidentiality. AH performed the data entry and analyzed the results while ASA interpreted the results and $M B Q$ assisted the corresponding author.

\section{REFERENCES}

1. Memon WA, Khalid SE, Sharif I, Saulat S, Haider A, Ullah A, et al. The efficacy of JJ stent on stone free rate after extracorporeal shock wave lithotripsy: A retrospective study. Pak J Med Dent. 2021;10(2): 22-27. doi: 10.36283/PJMD10-2/005
2. Jessani S, Bux R, Jafar TH. Prevalence, determinants, and management of chronic kidney disease in Karachi, Pakistan - A community based cross-sectional study. BMC Nephrol. 2014;15(1):1-9. doi: 10.1186/14712369-15-90

3. Jendeberg J, Geijer H, Alshamari M, Cierzniak B, Lidén M. Size matters: The width and location of a ureteral stone accurately predict the chance of spontaneous passage. Eur Radiol. 2017;27(11):4775-4785. doi: 10.1007/s00330-017-4852-6

4. Taguchi K, Cho SY, Ng ACF, Usawachintachit M, Tan YK, Deng YL, et al. The Urological Association of Asia clinical guideline for urinary stone disease. Int J Urol. 2019;26(7):688-709. doi: 10.1111/iju.13957

5. Binbay M, Tepeler A, Singh A, Akman T, Tekinaslan E, Sarilar $O$, et al. Evaluation of pneumatic versus holmium:YAG laser lithotripsy for impacted ureteral stones. Int Urol Nephrol. 2011;43(4):989-995. doi: 10.1007/s1 12 55-011-9951-8

6. Takazawa R, Kitayama S, Tsujii T. Successful outcome of flexible ureteroscopy with holmium laser lithotripsy for renal stones 2cm or greater. Int J Urol. 2012;19(3):264-267. doi: 10.1111/j.1442-2042.2011.02931.x

7. Degirmenci T, Gunlusoy B, Kozacioglu Z, Arslan M, Koras $O$, Arslan B, et al. Comparison of Ho:YAG laser and pneumatic lithotripsy in the treatment of impacted ureteral stones: An analysis of risk factors. Kaohsiung J Med Sci. 2014;30(3):153-158. doi: 10.1016/j.kjms. 2013.08.007

8. Becker B, Gross AJ, Netsch C. Ho:YaG laser lithotripsy: Recent innovations. Curr Opin Urol. 2019;29(2):103-107. doi: 10.1097/MOU.0000000000000573

9. Joshi A, Akhlaque M, Shahzad I, Imtiaz S, Khan S. Efficacy of pneumatic lithoclast in the management of upper ureteric stones. Pak J Med Heal Sci. 2020; 14(4):1 11 13-1116. doi: 10.26717/BJSTR.2018.02.000 813

10. Manohar T, Ganpule A, Desai M. Comparative evaluation of Swiss LithoClast $2^{\circledR}$ and Holmium:YAG laser lithotripsy for impacted upper-ureteral stones. J Endourol. 2008;22(3):443-436. doi: 10.1089/end.2007.0288

11. Kızllay F, Kalemci S, Turna B, Şimşir A, Nazlı O. Ho:YAG laser versus pneumatic lithotripsy for management of pediatric ureteral stones: a prospective-comparative analysis with adults. J Pediatr Urol. 2020;16(1):35.e1-35.e7. doi: 10.1016/j.jpurol.2019.11.006

12. Yin $X$, Tang $Z$, $Y \cup B$, Wang $Y$, Li $Y$, Yang $Q$, et al. Holmium: YAG laser lithotripsy versus pneumatic lithotripsy for treatment of distal ureteral calculi: A meta-analysis. J Endourol. 2013;27(4):408-414. doi: 10.1089/end.2012.0324

13. Panthier F, Ventimiglia E, Berthe L, Chaussain C, Daudon M, Doizi S, et al. How much energy do we need to ablate $1 \mathrm{~mm}^{3}$ of stone during Ho:YAG laser lithotripsy? An in vitro study. World J Urol . 2020;38(1 1):2945-2953. doi: 10.1007/s00345-020-03091-5

14. Chen S, Zhou L, Wei T, Luo D, Jin T, Li H, et al. Comparison of Holmium: YAG Laser and pneumatic lithotripsy in the treatment of ureteral stones: An update meta-analysis. Urol Int. 2017;98(2):125-133. doi: 


\section{$10.1159 / 000448692$}

15. Martov AG, Ergakov D V, Guseynov M, Andronov AS, Plekhanova OA. Clinical comparison of super pulse thulium fiber laser and high-power holmium laser for ureteral stone management. J Endourol. 2021;35(6):795-800. doi: 10.1089/end.2020.0581

16. Traxer $O$, Keller EX. Thulium fiber laser: the new player for kidney stone treatment? A comparison with Holmium:YAG laser. World J Urol. 2020;38(8):1883-1894. doi: 10.1007/s00345-019-02654-5

17. Sajid MT, Ameen M, Murtaza B, Alvi MS, Khan Z, Kiani F. Comparison of mean operative time in patients undergoing ho: YAG laser lithotripsy and pneumatic lithotripsy in ureterorenoscopy for ureteric calculus. Pak J Med Sci. 2021;37(2): 37(2): 415-420. doi: 10.12669/pjms.37.2.3049

18. ljaz I. Efficacy of pneumatic lithoclast in the management of urolithiasis in upper, middle and lower ureter. Biomed J Sci Tech Res. 2018;2(5):2864-2867. doi: 10.26717/BJSTR.2018.2.000813

19. MS Majeed M, Y Al-Timary A, A Mohammed M. Evaluation of pneumatic lithoclast efficacy in treatment of ureteric stones. Med J Basrah Univ. 2008;26(1):15-18. doi: 10.33762/mjbu.2008.48326

20. Rashid AO, Attar A, Mohammed KS, Fakhralddin SS, Abdulla LN, Buchholz N. Direct comparison of pneumatic and Ho:YAG laser lithotripsy in the management of lower ureteric stones. Urol Int. 2020;104(9-10):765-768. doi: 10.1159/000508419

21. Ficarra V, Novara G, Giannarini G, De Nunzio C, Abrate A, Bartoletti R, et al. Urology practice during the covid-19 vaccination campaign. Urol J. 2021; 88(4):298-305. doi: 10.1177/03915603211016321

22. Rabani SM, Rabani S, Rashidi N. Laser versus pneumatic lithotripsy with semi-rigid ureteroscope; A comparative randomized study. J Lasers Med Sci. 2019;10(3):185-188. doi: 10.15171/jlms.2019.29

23. Liu C, Zhou H, Jia W, Hu H, Zhang H, Li L. The Efficacy of percutaneous nephrolithotomy using pneumatic lithotripsy vs. the holmium laser: A randomized study. Indian J Surg. 2017;79(4):294-298. doi: 10.1007/s1226 2-016-1473-2

24. Kassem A, Elfayoumy $H$, Elsaied W, Elgammal $M$, Bedair A. Laser and pneumatic lithotripsy in the endoscopic management of large ureteric stones: A comparative study. Urol Int. 2012;88(3):311-315. doi: $10.1159 / 000336254$

25. Zyczkowski M, Bogacki R, Nowakowski K, Muskała B, Rajwa P, Bryniarski P, et al. Application of pneumatic lithotripter and holmium laser in the treatment of ureteral stones and kidney stones in children. Biomed Res Int. 2017;2017(1):1-7. doi: 10.1155/2017/2505034. 